JOURNAL OF SYNCHROTRON RADIATION

ISSN 1600-5775

Received 11 January 2021

Accepted 14 April 2021

Edited by S. M. Heald, Argonne National Laboratory, USA

Keywords: XLD; STXM; soft X-rays; ZnO.

\section{Soft X-ray nanospectroscopy for quantification of X-ray linear dichroism on powders}

\author{
Selwin Hageraats, ${ }^{a, b, c *}$ Mathieu Thoury, ${ }^{b}$ Stefan Stanescu ${ }^{d}$ and Katrien Keune ${ }^{a, c}$ \\ ${ }^{a}$ Conservation and Science, Rijksmuseum, PO Box 74888, 1070DN Amsterdam, The Netherlands, bIPANEMA, CNRS, \\ Ministère de la Culture et de la Communication, Université de Versailles Saint-Quentin-en-Yvelines, USR 3461, \\ Université Paris-Saclay, 91128 Gif-sur-Yvette, France, ' $V a n$ 't Hoff Institute for Molecular Science, University of \\ Amsterdam, PO Box 94157, 1090 GD Amsterdam, The Netherlands, and ${ }^{\mathbf{d}}$ Synchrotron SOLEIL, 91192 Gif-Sur-Yvette \\ Cedex, France. *Correspondence e-mail: selwin.hageraats@wur.nl
}

X-ray linear dichroism (XLD) is a fundamental property of many ordered materials that can for instance provide information on the origin of magnetic properties and the existence of differently ordered domains. Conventionally, measurements of XLD are performed on single crystals, crystalline thin films, or highly ordered nanostructure arrays. Here, it is demonstrated how quantitative measurements of XLD can be performed on powders, relying on the random orientation of many particles instead of the controlled orientation of a single ordered structure. The technique is based on a scanning X-ray transmission microscope operated in the soft $\mathrm{X}$-ray regime. The use of a Fresnel zone plate allows X-ray absorption features to be probed at $\sim 40 \mathrm{~nm}$ lateral resolution $-\mathrm{a}$ scale small enough to probe the individual crystallites in most powders. Quantitative XLD parameters were then retrieved by determining the intensity distributions of certain diagnostic dichroic absorption features, estimating the angle between their transition dipole moments, and fitting the distributions with four-parameter dichroic models. Analysis of several differently produced $\mathrm{ZnO}$ powders shows that the experimentally obtained distributions indeed follow the theoretical model for XLD. Making use of Monte Carlo simulations to estimate uncertainties in the calculated dichroic model parameters, it was established that longer X-ray exposure times lead to a decrease in the amplitude of the XLD effect of $\mathrm{ZnO}$.

\section{Introduction}

The X-ray absorption behavior of materials at and above elemental absorption edges can provide a wealth of information on oxidation states, coordination symmetry, and distortions in the coordination environment. Depending on the type of information that is required, one may either probe the discrete bound-to-bound transitions directly around the absorption edge (X-ray absorption near-edge structure, abbreviated XANES) or the bound-to-continuum oscillations further above the absorption edge (extended X-ray absorption fine structure, abbreviated EXAFS). When studying ordered materials, such as crystals or certain polymers, X-ray absorption spectra recorded with linearly polarized X-rays may exhibit a distinct angle-dependency. That is, the intensities of some absorption features fluctuate upon rotating the ordered structures relative to the axis of polarization. This effect is called X-ray linear dichroism (XLD) and can either be related to anisotropies in the electronic or the magnetic properties of materials. This first property is called X-ray natural linear dichroism (XNLD) and occurs due to charge anisotropies in certain ordered materials. Such ordered materials can either be crystalline, in which case they have a non-cubic crystal 
structure with a rotation axis of order three or higher (Brouder, 1990), or non-crystalline, in which case the material has for instance formed non-covalent ordered structures of low symmetry (e.g. molecular adsorbates) (Zharnikov \& Neuber, 2000; Fu \& Urquhart, 2005). The second property is called X-ray magnetic linear dichroism (XMLD) and occurs in crystals that possess a preferential magnetic axis such as in ferromagnetic and antiferromagnetic materials (Thole et al., 1985; Schwickert et al., 1998; Kortright \& Kim, 2000; Kuiper et al., 1993; Spanke et al., 1998; Antel Jr et al., 1999; Scholl et al., 2000). The study of these two properties can provide information on the configuration of impurities in host lattices (Gaudry et al., 2005; Schauries et al., 2013), the magnetic characteristics of materials (Spanke et al., 1998; Antel Jr et al., 1999; Scholl et al., 2000) and the existence of order in organic systems (Zharnikov \& Neuber, 2000; Smith \& Ade, 1996).

Experimentally, XLD is typically studied by rotating a sample with respect to the electric field vector of the polarized $\mathrm{X}$-rays or vice versa. For each angle, an X-ray absorption spectrum or the absorption at a few characteristic features is recorded. For this set-up to work, it is crucial that within the measured sample volume all material has the same spatial order. This typically requires either a single crystal (Gaudry et al., 2005; Juhin et al., 2008), a crystalline thin film (Spanke et al., 1998; Scholl et al., 2000), a highly ordered array of nanostructures (Chiou et al., 2004) or an ordered ensemble of molecular adsorbates (Zharnikov \& Neuber, 2000).

Alternatively, in macroscopically disordered materials, the spatial distribution of differently oriented domains can be spatially resolved using scanning transmission X-ray microscopy (STXM) or X-ray photoelectron emission microscopy (XPEEM) in combination with linearly polarized X-ray beams (Spanke et al., 1998; Scholl et al., 2000; Chiou et al., 2004; Smith \& Ade, 1996). In STXM, the X-ray beam is focused down to a spot size of the order of tens of nanometres and raster scanned across a sample. The transmitted X-rays are recorded per point and can be reconstructed to a sample image. XPEEM, on the other hand, makes use of the photoelectric effect with linearly polarized X-rays tuned to be in resonance with core electron binding energies. Using either a tightly focused beam (then called scanning X-ray photoelectron microscopy, abbreviated SPEM) or full-field irradiation, XLD images can be obtained with a spatial resolution in the order of tens of nanometres.

It is proposed here to take advantage of the extraordinarily high spatial resolution of STXM to quantify XLD properties on powders - hereby circumventing the need for single crystals or other highly ordered sample structures. The proposed technique makes use of the random orientation of many crystallites to obtain distributions whose width and skewness hold information on the XLD parameters. Recognizing the quadratic dependency of the absorption crosssection on the amplitude of the electromagnetic perturbation following Fermi's golden rule (Stöhr, 1992), and the dependency of the perturbation on the projection of the polarization vector e onto the transition dipole moment, the following model for XLD is used,

$$
\sigma=\sigma_{0}+\sigma_{1}\left|\mathbf{e} \cdot\left\langle\psi_{\mathrm{f}}|\mathbf{p}| \psi_{\mathrm{i}}\right\rangle\right|^{2}
$$

where $\sigma$ is the absorption cross-section for a transition between initial state $\psi_{\mathrm{i}}$ and final state $\psi_{\mathrm{f}}, \mathbf{p}$ is the electron momentum operator, and $\sigma_{0}$ and $\sigma_{1}$ are the relative amplitudes of the angle-independent and angle-dependent fractions of the absorption cross-section. Writing $\psi_{\mathrm{f}}|\mathbf{p}| \psi_{\mathrm{i}}$ as the transition dipole moment vector $\mu_{\mathrm{if}}$, one obtains

$$
\sigma=\sigma_{0}+\sigma_{1}\left|\mathbf{e} \cdot \boldsymbol{\mu}_{\mathrm{if}}\right|^{2}
$$

which can be expressed as a function of the angle $\delta$ between the polarization vector and transition dipole moment according to

$$
\sigma=\sigma_{0}+\sigma_{1}\|\mathbf{e}\|\left\|\boldsymbol{\mu}_{\mathrm{if}}\right\| \cos ^{2} \delta=\sigma_{0}+\sigma_{1}^{\prime} \cos ^{2} \delta .
$$

Transforming to computationally more straightforward polar coordinates and defining the polarization vector e to have coordinates $(1, \vartheta, \varphi)=(1,0, \pi / 2)$, one obtains

$$
\sigma=\sigma_{0}+\sigma_{1}^{\prime} \cos ^{2} \vartheta \sin ^{2} \varphi .
$$

To demonstrate how XLD can be quantified on powders, a proof-of-principle study is described here on $\mathrm{ZnO}$ : a semiconductor that has been shown previously to exhibit XNLD based on $\mathrm{Zn} K$-edge and $\mathrm{O} K$-edge studies of $\mathrm{ZnO}$ single crystals and thin $\mathrm{ZnO}$ epilayers, respectively (Goulon et al., 2007; Preston et al., 2008). Here, XNLD is studied at the Zn Ledge $(\sim 1030 \mathrm{eV})$ in order to facilitate compatibility with a soft $\mathrm{X}$-ray STXM at the highest possible photon flux. Although this study quantifies XNLD, the mathematical similarity with XMLD means that such dichroic parameters could be similarly quantified using the proposed methodology.

This work is based in part on the experimental data presented first by Hageraats et al. (2020), and offers a revised interpretation of the results.

\section{Methods}

\subsection{Sample preparation}

Samples from four different batches of $\mathrm{ZnO}$ were analyzed. Three of these were zinc white pigments produced using the French (indirect) vapor oxidation process and were obtained from modern pigment manufacturers. The three zinc white samples are henceforth referred to as FrMod1-3. A fourth sample of ultrapure $\mathrm{ZnO}$ was lab-synthesized by combusting high-purity Zn-foil (99.99\%, $0.125 \mathrm{~mm}$ thickness; Advent Research Materials Ltd) in a stainless steel airtight glovebox. A controlled atmosphere was created by outgassing and purging with argon three consecutive times and then introducing an 80/20 mixture of $\mathrm{Ar} / \mathrm{O}_{2}$ (Air Liquide, $\mathrm{Ar}>99.99 \%$ and $\mathrm{O}_{2}>99.995 \%$ purity) into the glovebox up to a pressure of 1 bar. After combustion, the ultrapure $\mathrm{ZnO}$ powder was collected in the inert atmosphere on a glass plate. X-ray photoelectron spectroscopy (XPS) analysis of the labsynthesized batch showed no detectable impurities. This sample is henceforth referred to as $\mathrm{ZnO}-\mathrm{L}$.

All four $\mathrm{ZnO}$ samples were prepared for STXM analysis by suspending a few hundred $\mu \mathrm{g}$ of $\mathrm{ZnO}$ in $\sim 1 \mathrm{ml}$ of isopropanol, 
sonicating the suspension for several minutes and then drop casting a $\sim 5 \mu \mathrm{L}$ droplet onto a $100 \mathrm{~nm}$ thin $\mathrm{SiN}$ window heated to $\sim 100^{\circ} \mathrm{C}$. After several seconds, all isopropanol evaporated, leaving small amounts of $\mathrm{ZnO}$ dispersed over the window surface.

\subsection{Scanning transmission X-ray microscopy}

STXM experiments were conducted at the HERMES beamline of the SOLEIL synchrotron. The undulator radiation was monochromated with a monochromator that supports transmission of photons up to $1600 \mathrm{eV}$. The STXM (Research Instruments $\mathrm{GmbH}$ ) was equipped with a $30 \mathrm{~nm}$ outer ring width Fresnel zone plate (FZP) and $50 \mu \mathrm{m}$ order-sorting aperture to focus the monochromated beam down to a diameter of approximately $40 \mathrm{~nm}$. SiN windows supporting the various $\mathrm{ZnO}$ samples were fixed to an $X Y Z$ scanning stage, $Z$ being the direction collinear with the X-ray beam propagation and used to bring the sample onto the FZP focal plane. STXM images were obtained by raster scanning the $X Y$ position and recording the transmitted photons using a photomultiplier tube (PMT). Based on low-resolution overview images of the $\mathrm{SiN}$ windows, the $\mathrm{ZnO}$ dispersions were found to consist of numerous clusters of several tens to hundreds mostly unstacked $\mathrm{ZnO}$ crystallites - each cluster measuring several $\mu \mathrm{m}$ in diameter. Therefore, the dimensions of the STXM images were set to be in the range $3 \mu \mathrm{m} \times 3 \mu \mathrm{m}$ to $6 \mu \mathrm{m} \times 6 \mu \mathrm{m}$. XANES contrast maps were obtained on all four samples (FrMod1-3, ZnO-L) by recording $\sim 150$ consecutive images at X-ray energies in the range $1015-1050 \mathrm{eV}$, thereby obtaining hyperspectral $\mathrm{Zn} L$-edge maps. In addition, the FrMod1 and $\mathrm{ZnO}-\mathrm{L}$ samples were analyzed by choosing just two diagnostic energies (1031 and $1033 \mathrm{eV}$ ) that show the strongest XNLD behavior, hereby minimizing the effect of possible radiation damage due to prolonged exposure to the high-flux X-ray beam and maximizing the number of spatial points that can be analyzed in a reasonable timeframe.

\subsection{Data processing}

2.3.1. Visualizing XNLD contrast. STXM energy stacks first were aligned using a Fourier alignment algorithm and transformed from transmission to optical density (OD) in the aXis2000 software. XNLD contrast was visualized in the hyperspectral STXM-XANES maps by running the simplex volume maximization (SiVM) endmember selection algorithm (adapted from the DataHandlerP software) (Thurau et al., 2012) and fitting the data with non-negative least-squares (NNLS) using the first four endmembers as input factors. Following the SiVM approach, endmember spectra are individual spectra from the dataset which, in the most ideal case, are thought to constitute the purest representations of the different compounds present in the sample. In this ideal case, the endmembers of a pure and dichroic sample can be interpreted as those spectra exhibiting the largest dichroic differences. Following the initial SiVM endmember selection, the endmembers were updated by averaging all spectra scoring primarily high on endmembers 1 and 4, chosen based on their low degree of covariance. The resulting endmembers [see Fig. 1(a)] were found to represent two distinctly different e $\cdot \boldsymbol{\mu}_{\text {if }}$ projections. A second NNLS fit with the two updated endmembers then yielded weight matrices that were used as red and blue channels to produce false color $R(G) B$ images showing the XNLD contrast among individual $\mathrm{ZnO}$ crystallites in the four hyperspectral Zn L-edge STXM datasets.

2.3.2. Quantifying XNLD. From the two dichroic spectral endmembers, two diagnostic energies were determined. These absorption features were found to respond most strongly and oppositely - as a function of the projection angle $\delta$, making them suitable for quantification of the XNLD parameters. As the authors discussed in a previous paper on soft
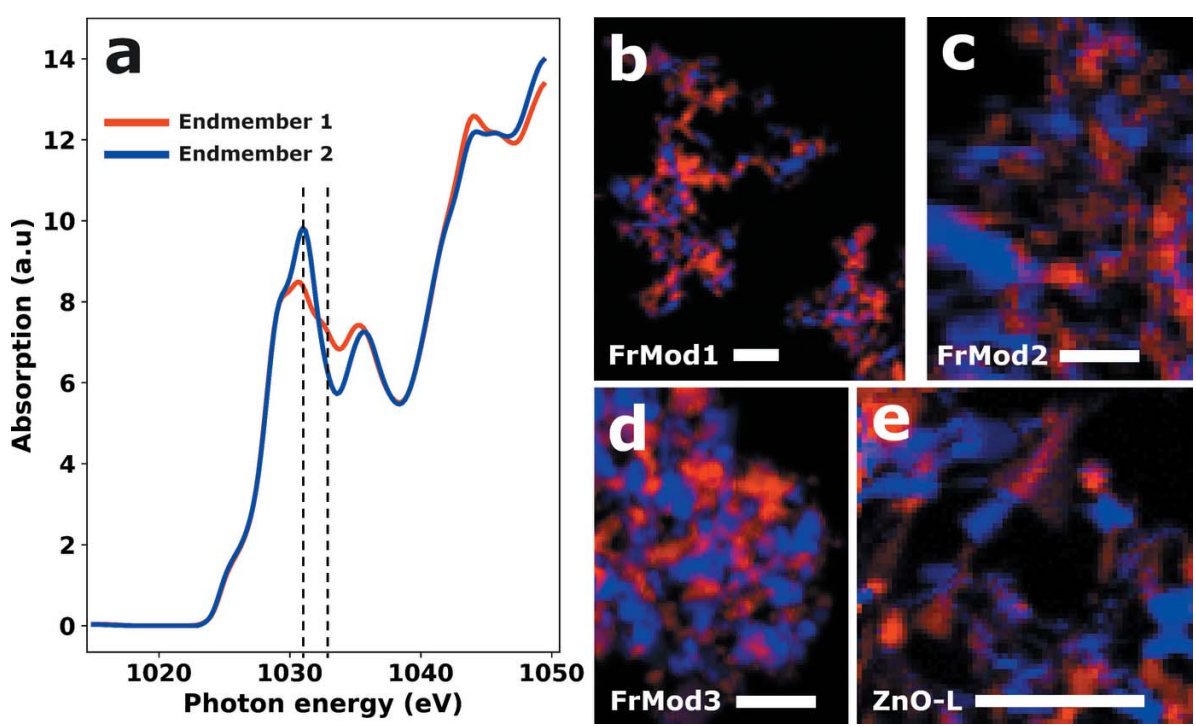

Figure 1

SiVM-NNLS analysis of the four hyperspectral STXM data sets. (a) Plot of endmembers 1 and 2. ( $b-e$ ) False color representations of the NNLS fits with endmembers 1 (red channel) and 2 (blue channel) of samples FrMod1-3 and ZnO-L. All scale bars have a length of $1 \mu \mathrm{m}$. 


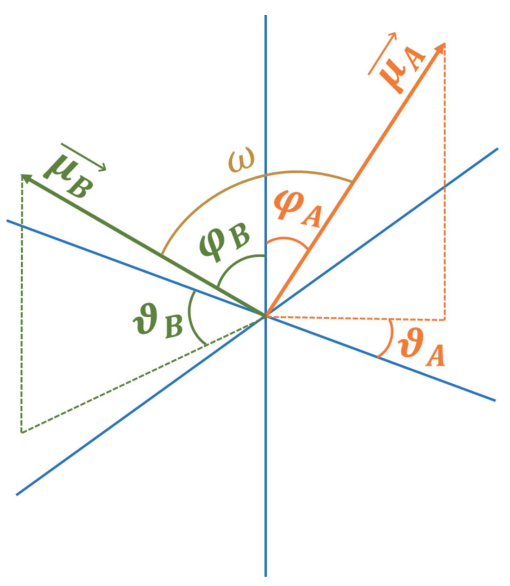

Figure 2

Illustration of the angular coordinates of transition dipole moments $\overrightarrow{\mu_{A}}$ and $\overrightarrow{\mu_{B}}$, corresponding to the transitions at the two diagnostic energies.

$\mathrm{X}$-ray nanospectroscopy of $\mathrm{ZnO}$, these two diagnostic energies are thought to correspond to transitions between $\mathrm{Zn} 2 p(J=$ $3 / 2$ ) and $\mathrm{Zn} 4 d$ derived states (Hageraats et al., 2020). The anisotropic character of the $\mathrm{Zn} 4 d$ derived states causes both these transitions to have a dichroic character. Furthermore, the different symmetries and/or angular offset among different $\mathrm{Zn} 4 d$ derived states mean that the XLD model proposed in equation (4) needs to be extended to include a phase shift $\omega$. This angle $\omega$ represents the angle between the transition dipole moments for these two diagnostic transitions. The complete model for two symmetrically distinct transitions $A$ and $B$ then becomes

$$
\begin{aligned}
& \sigma_{A}=\sigma_{A, 0}+\sigma_{A, 1}^{\prime} \cos ^{2} \vartheta_{A} \sin ^{2} \varphi_{A}, \\
& \sigma_{B}=\sigma_{B, 0}+\sigma_{B, 1}^{\prime} \cos ^{2} \vartheta_{B} \sin ^{2} \varphi_{B},
\end{aligned}
$$

with

$$
\frac{\mathbf{C}\left(1, \vartheta_{A}, \varphi_{A}\right) \cdot \mathbf{C}\left(1, \vartheta_{B}, \varphi_{B}\right)}{\left\|\mathbf{C}\left(1, \vartheta_{A}, \varphi_{A}\right)\right\|\left\|\mathbf{C}\left(1, \vartheta_{B}, \varphi_{B}\right)\right\|}=\cos \omega
$$

where the operator $\mathbf{C}$ indicates transformation of the vector from spherical coordinates to Cartesian coordinates. Fig. 2 illustrates the relation between the two transition dipole moments $\overrightarrow{\mu_{A}}$ and $\overrightarrow{\mu_{B}}$, with all angles indicated.

In order to link the model to the experimental data, all spectra were normalized to their integral. This corrects for the local OD and yields values that are approximately proportional to the absorption cross-section. Since relative $\sigma$ values provide sufficient information to quantitatively compare the XNLD behavior of different powders, the proportionality factor is here assumed to be unity, simply giving $\sigma_{A}=I_{A}$ and $\sigma_{B}=I_{B}$, where $I_{A}$ and $I_{B}$ are the experimentally measured and normalized intensities at the two diagnostic energies 1031 and $1033 \mathrm{eV}$, respectively. Assuming no significant overlap between the two absorption features, the model for the experimental data then becomes

$$
\begin{gathered}
I_{A}=\sigma_{A, 0}+\sigma_{A, 1}^{\prime} \cos ^{2} \vartheta_{A} \sin ^{2} \varphi_{A}+\varepsilon_{A}, \\
I_{B}=\sigma_{B, 0}+\sigma_{B, 1}^{\prime} \cos ^{2} \vartheta_{B} \sin ^{2} \varphi_{B}+\varepsilon_{B},
\end{gathered}
$$

with the relation between $\vartheta_{A}, \varphi_{A}, \vartheta_{B}, \varphi_{B}$ still dictated according to equation (7). Here, $\varepsilon_{A}$ and $\varepsilon_{B}$ are experimental errors that are here both assumed to be normally distributed around zero with an expected absolute value $|\varepsilon|$. Experimental distributions were determined by binning all values of $I_{A}$ and $I_{B}$ from a certain dataset in 30 bins.

Model distributions were determined by picking approximately $10^{5}$ pairs of random unit vectors that represent the two transitions dipole moments of $\sim 10^{5}$ individual crystallites. According to a procedure laid out in Fig. 3, each first vector of the random vector pairs $\left(\mathbf{v}_{1}\right)$ was determined by randomly picking $x, y$, and $z$ coordinates from a single Gaussian distribution and normalizing its length to unity, yielding vectors whose coordinates are uniformly distributed on a unit sphere (Muller, 1959). Each second vector $\left(\mathbf{v}_{2}\right)$ is picked by recognizing that all vectors under an angle $\omega$ with respect to the first vector exist on a cone with projection $\cos \omega$ and an azimuthal angle $\psi$. Therefore, each second vector can be picked by randomly sampling an angle $\psi$ from a uniform distribution $[0,2 \pi]$ - yielding polar coordinates $(1, \psi, \omega)$ - determining its Cartesian coordinates in a coordinate system with $\mathbf{v}_{1}$

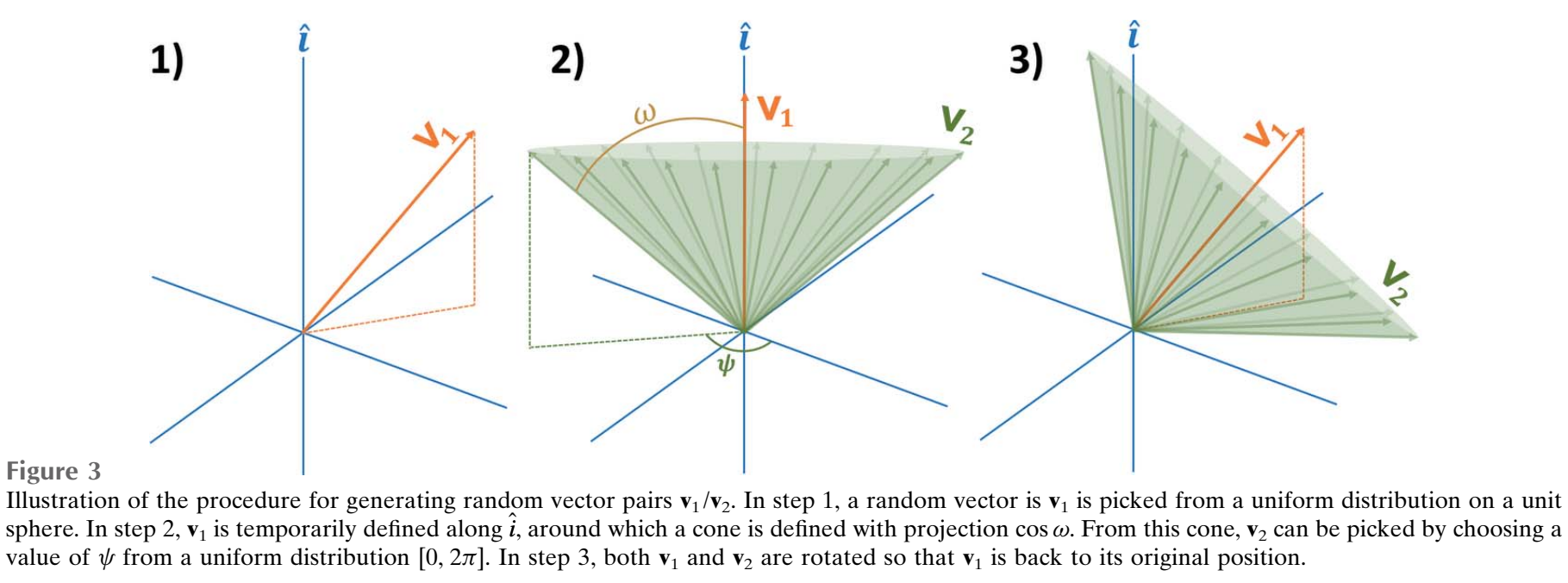


pointing along $\hat{i}$, and rotating the vector using Rodrigues' rotation formula,

$$
\mathbf{v}_{2}=\mathbf{v}_{2}^{\prime} \cos \delta+\left(\mathbf{k} \times \mathbf{v}_{2}^{\prime}\right) \sin \delta+\mathbf{k}\left(\mathbf{k} \cdot \mathbf{v}_{2}^{\prime}\right)(1-\cos \delta),
$$

where $\mathbf{v}_{2}^{\prime}=(\cos \psi \sin \omega, \sin \psi \sin \omega, \cos \omega)$ is the randomly picked second vector of the vector pair prior to rotation, $\mathbf{k}=$ $\left(\hat{i} \times \mathbf{v}_{1}\right)$ is the axis of rotation, and $\delta=\arccos \left(\hat{i} \cdot \mathbf{v}_{1}\right)$ is the rotation angle.

These sets of randomly oriented unit vectors can then be plugged into equations (8) and (9) to obtain model distributions of $I_{A}$ and $I_{B}$ for given dichroic model parameters $\sigma_{A, 0}$, $\sigma_{A, 1}^{\prime} \sigma_{B, 0}$, and $\sigma_{B, 1}^{\prime}$ and expected experimental error $|\varepsilon|$. Quantification of the dichroic model parameters and experimental error for given experimental distributions can then be performed by applying a fitting algorithm.

Central to the fitting algorithm is a cost function $F_{\mathrm{C}}$ that measures the goodness of fit of a model distribution [equations (8) or (9)] with respect to an experimental distribution. $F_{\mathrm{C}}$ takes as input a set of dichroic model parameters $\left(\sigma_{A, 0}, \sigma_{A, 1}^{\prime}, \sigma_{B, 0}, \sigma_{B, 1}^{\prime}\right)$, an expected experimental error $(|\varepsilon|)$, and a pre-calculated set of random unity vectors. It then calculates a model distribution, compares it with the experimental distribution, and returns the sum of squared errors. Optimization of the model parameters and estimation of the expected experimental error was done by means of a tailored gradient descent method. The gradient descent method works by calculating the gradient of the cost function $F_{\mathrm{C}}$ around an estimate $\mathbf{P}_{n}=\left(\sigma_{A, 0}, \sigma_{A, 1}^{\prime}, \sigma_{B, 0}, \sigma_{B, 1}^{\prime},\langle|\varepsilon|\rangle\right)$ and updating the estimation according to

$$
\mathbf{P}_{n+1}=\mathbf{P}_{n}-\lambda \nabla F_{\mathrm{C}}\left(\mathbf{P}_{n}\right),
$$

where $\lambda$ is a constant that is manually chosen so as to ensure fast and reliable convergence onto local minima. The parameter vector $\mathbf{P}_{n}$ that produces a global minimum of the cost function $F_{\mathrm{C}}$ is regarded as the best approximation of the experimental distribution.

To facilitate the estimation of a realistic uncertainty in the calculated dichroic model parameters, a Monte Carlo simulation of synthetic datasets was performed for each experimental distribution. Synthetic datasets of experimental distributions $I_{A}$ and $I_{B}$ were calculated by assuming that the estimates of the dichroic model parameters and experimental error - obtained through the fitting algorithm - are close to the true values. Accurate synthetic copies of the experimental distributions can then be obtained by picking a number of vector pairs that is equal to the number of measurements of $I_{A}$ and $I_{B}$, with a number of unique orientations equal to the number of crystallites captured in the experimental dataset. Each vector pair is then evaluated according to equations (8) and (9), yielding two synthetic distributions. Each synthetic dataset was modeled using the previously described fitting algorithm, yielding multiple estimates of each dichroic model parameter. The standard deviation among these estimates was taken as $u_{P, \text { exp }}$ : the uncertainty in an estimate of model parameter $P$ due to the limited number of measurements that constitute the experimental datasets.

\section{Results}

To give an idea of the extent and the nature of the XNLD of $\mathrm{ZnO}$ powders, Figs. 1(b)-1(e) shows false-color XNLD images of the samples FrMod1-3 and ZnO-L determined from NNLS fits of the hyperspectral STXM datasets with the endmembers shown in Fig. 1(a). Distributions of $\mathrm{ZnO}$ crystallites can be seen that exhibit roughly $50 \%$ red and $50 \%$ blue particles, meaning their orientation with respect to the polarization axis is either most similar to that of endmember 1 or that of endmember 2 . The ability to nearly binarily classify particles as either red or blue means that the two endmembers do not represent extremities in the $\mathbf{e} \cdot \boldsymbol{\mu}_{\text {if }}$ projections but rather intermediaries. The true extent of the XNLD effect in $\mathrm{ZnO}$ therefore must exceed the effect that separates endmembers 1 and 2.

Fig. 4 shows the experimental distributions of $I_{A}$ and $I_{B}$, grouped together based on whether the data is obtained from a hyperspectral STXM energy stack [Figs. 4(a) and 4(b)], a two-energy STXM stack [Figs. $4(c)$ and $4(d)$ ], only the FrMod3 sample [Figs. 4(e), and 4(f)], or only the ZnO-L sample [Figs. $4(g)$ and $4(h)$ ]. From these distributions, it can clearly be surmised that the distributions obtained from hyperspectral STXM energy stacks are narrower than those obtained from just two energies. Furthermore, the $I_{B}$ distribution obtained from a two-energy STXM stack recorded on the ZnO-L sample appears to deviate significantly from the same distribution obtained on the FrMod3 sample [Fig. 4(d)].

In order to quantify these visually observed differences in terms of dichroic model parameters, it is first necessary to estimate the angle $\omega$. Although this angle does partly dictate the relation between $I_{A}$ and $I_{B}$ [see equation (7)], random variations in $\varphi_{A}, \varphi_{B}$ and experimental noise weaken this relation to such an extent that it was found to be impossible to retrieve an accurate measure of $\omega$ through analysis of the raw data. Instead, an estimate was made based on the spectral endmembers shown in Fig. 1(a). Given these two distinctly different projections of $\boldsymbol{\mu}_{\text {if }}$ onto e, there appears to be a negative correlation, corresponding to a value of $\omega=\pi / 2$ for $a \cos ^{2} \vartheta$ relationship. In terms of the orbitals involved in the transitions related to the two diagnostic energies, this means that the orbitals of the two $\mathrm{Zn} 4 d$ derived states (see Section 2.3.2) have a $90^{\circ}$ angular offset.

This value was plugged into the model proposed in equations (8) and (9) to define the cost function central to the fitting algorithm. Convergence of the algorithm onto global minima was found to be problematic due to the noisy nature of the cost function. This property can be observed in Fig. 5, which shows the cost in fitting an experimental distribution $I_{A}$ as a function of dichroic parameters $\sigma_{B, 0}$ and $\sigma_{B, 1}^{\prime}$, evaluated around what is thought to be the global minimum. This noisy nature is most likely related to the fact that the model distributions are not continuous functions, but rather consist of bins that contain an inherently finite number of samples. Small changes in the model parameters may only shift a few samples to new bins, a process that is prone to introduce highfrequency shot noise to the cost function. 

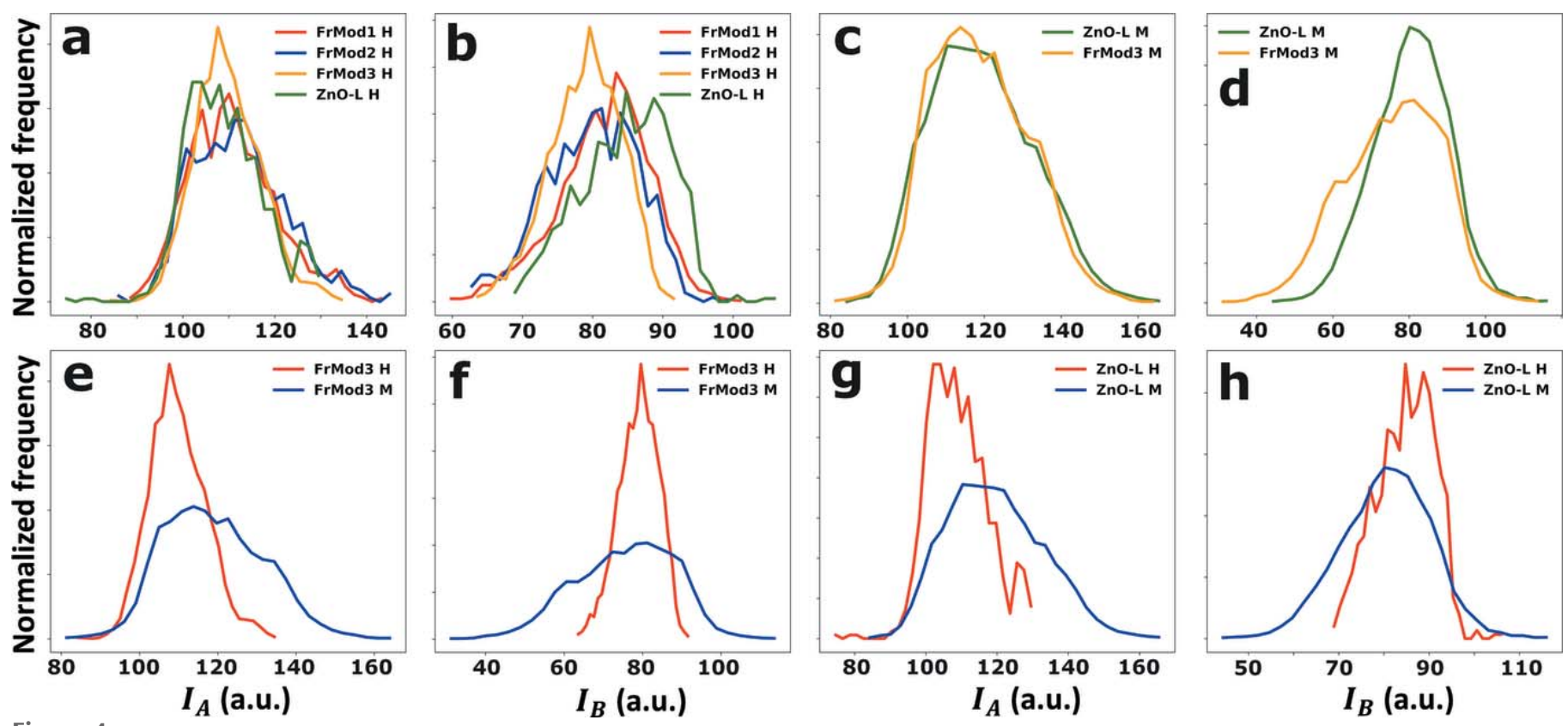

Experimental distributions of $I_{A}$ and $I_{B}$ for $(a, b)$ the hyperspectral data sets, $(c, d)$ the multispectral data sets, $(e, f)$ the data recorded on the FrMod3 sample, and $(g, h)$ the data recorded on the $\mathrm{ZnO}-\mathrm{L}$ samples.

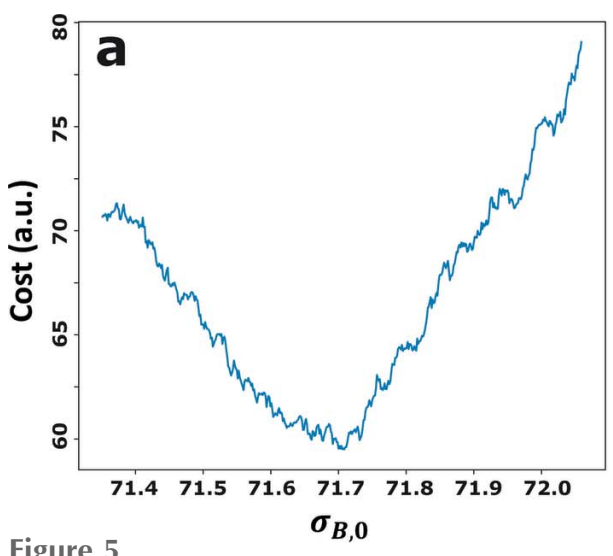

Figure 5

The cost of fitting an experimental distribution of $I_{A}$ around a minimum, evaluated as a function of dichroic model parameters $\sigma_{B, 0}(a)$ and $\sigma_{B, 1}^{\prime}(b)$.

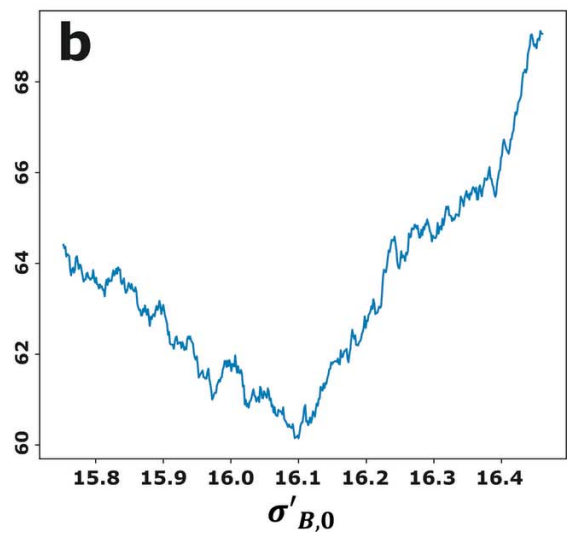

These noise characteristics were dealt with by introducing three modifications to the default gradient descent method. First, the parameter space over which local gradients were evaluated was set a lot bigger than usual, so as to take only the longer-range slopes into account and avoid sensitivity to noise. Second, as the cost function noise may still occasionally cause gross overestimation of the gradient and force the algorithm far away from the (local) minimum, the size of each optimization step was normalized to approximately $0.005 \sigma_{A, 0}$ for the first 20-50 iterations and approximately $0.001 \sigma_{A, 0}$ for all subsequent iterations. Third, due to the vast number of local minima, the algorithm was found to rarely converge on the same parameters twice for a given experimental or synthetic distribution. For this reason, after each convergence an initial guess was reinitialized by randomly mutating the dichroic parameters corresponding to the lowest previously found value of the cost function. Per 1000 iterations, the algorithm converged 5-15 times with a cost at most $25 \%$ higher than the absolute lowest cost value found. As a $25 \%$ difference in cost is not thought to be significant for data with noise levels this high, all of these convergences could be used together to estimate the dichroic parameters and determine $u_{P \text {, mod }}$ : the uncertainty in an estimate of model parameter $P$ due to the modeling error.

Fig. 6 shows the fits obtained for each of the experimental distributions, taking the dichroic parameters obtained for $\omega=\pi / 2$ and iterating the optimization algorithm 10000 times. It can be seen that the width and skew of the experimental distributions is mostly modeled accurately in the case of $I_{A}$, but less accurately in the case of $I_{B}$. To be precise, the experimental distributions of $I_{B}$ tend to have a negative skew, while the model only permits a positive skew.

This issue is illustrated in Fig. 7(a), where it shows the numerically approximated probability density function (PDF) of $\cos ^{2} \vartheta \sin ^{2} \varphi+\varepsilon$ for randomly oriented unit vectors with different levels of noise $\varepsilon$. The PDF clearly has a positive skew and can only be made (nearly) symmetrical by adding a high level of noise. One way in which a PDF with the right (negative) skew can be obtained is to add an out-of-phase contribution. Fig. 7(b) shows the PDFs of $\cos ^{2} \vartheta \sin ^{2} \varphi+$ $\alpha \cos ^{2} \vartheta^{\prime} \sin ^{2} \varphi^{\prime}+\varepsilon$ with every set of angles describing two perpendicular vectors: $\mathbf{C}(1, \vartheta, \varphi) \cdot \mathbf{C}\left(1, \vartheta^{\prime}, \varphi^{\prime}\right)=0$. Values of $\alpha$ were chosen between 0 and 1.3 and the noise level $|\varepsilon|$ was fixed at 0.15 . In Fig. 7(b) it can be seen that, when the in-phase and out-of-phase contributions become roughly equal, the 

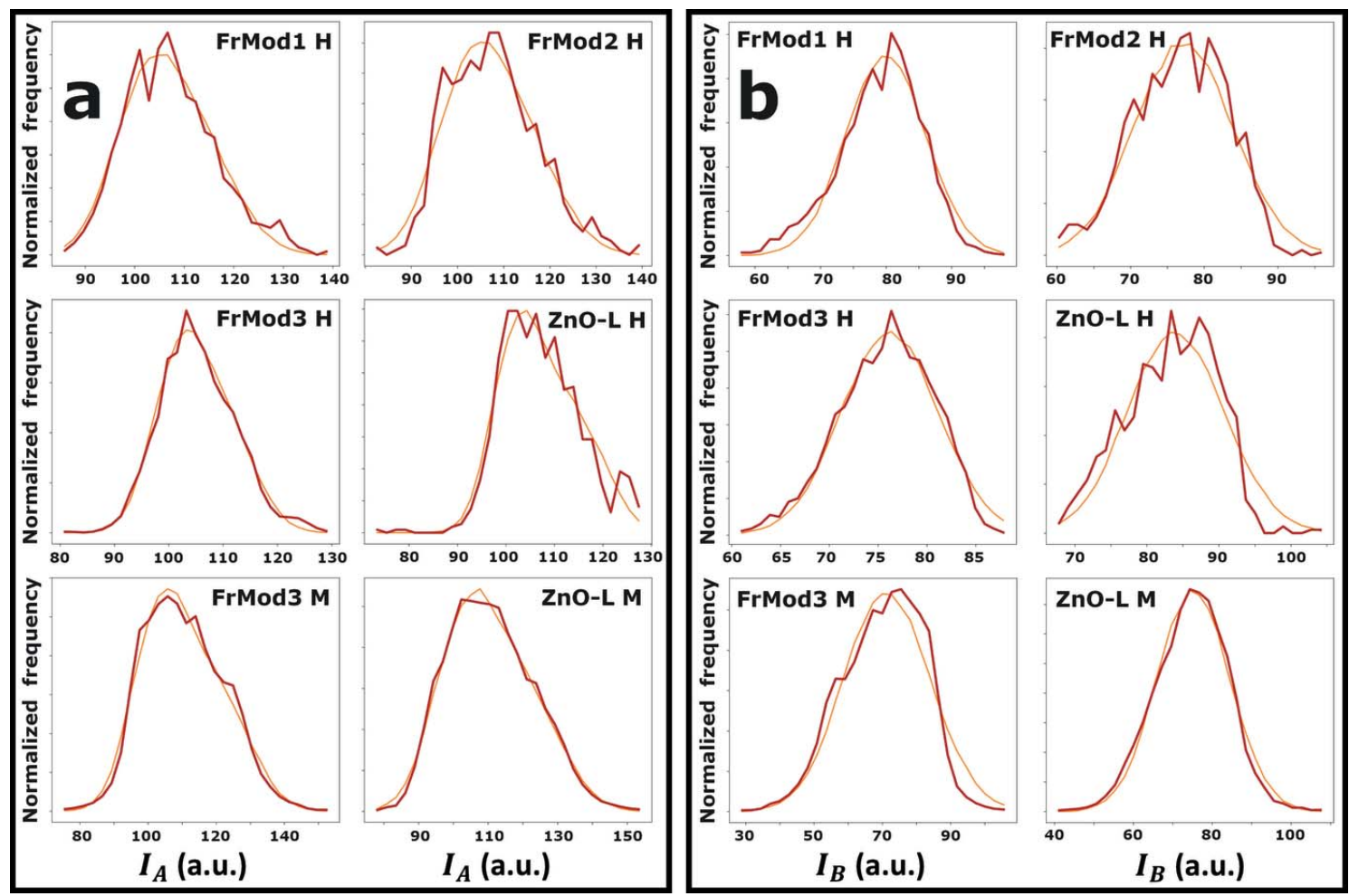

Figure 6

Experimental distributions (red) of $I_{A}(a)$ and $I_{B}(b)$ fitted according to the dichroic model postulated in equations (8) and (9) (orange).
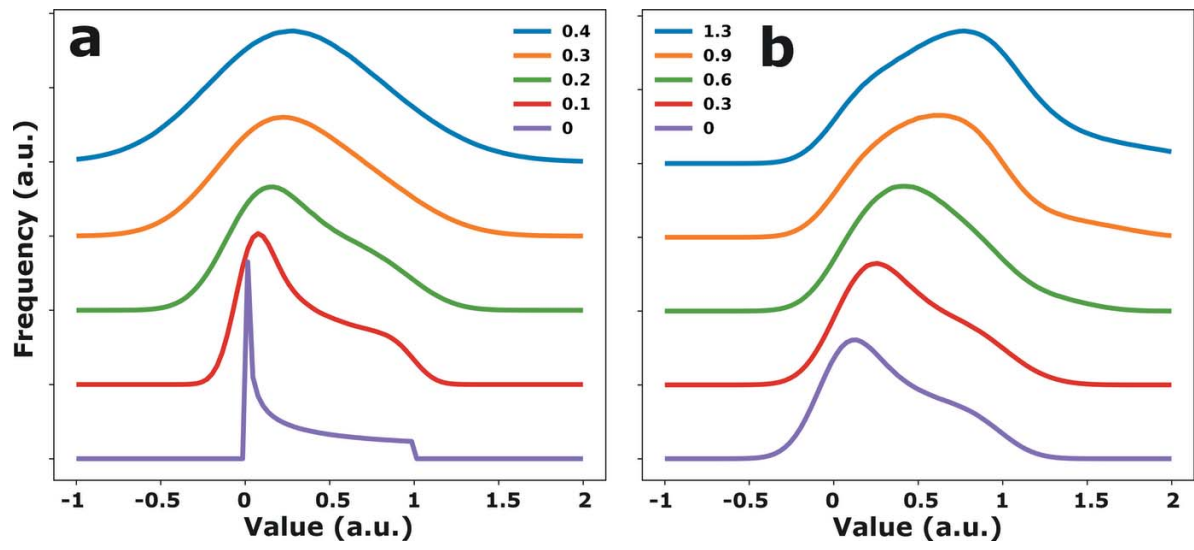

Figure 7

(a) PDFs of $\cos ^{2} \vartheta \sin ^{2} \varphi+\varepsilon$ evaluated for values of $\varepsilon$ ranging from zero (purple) to 0.4 (blue). (b) PDFs of $\cos ^{2} \vartheta \sin ^{2} \varphi+\alpha \cos ^{2}[\vartheta-(\pi / 2)] \sin ^{2} \varphi+\varepsilon$ evaluated for $\varepsilon=0.15$ and values of $\alpha$ ranging from 0 (purple) to 1.3 (blue).

skew becomes negative. With regard to the experimental distributions of $I_{B}$, this means that the intensity of the feature at $1033 \mathrm{eV}$ must have contributions from two different transitions with transition dipole moments oriented at a large angle relative to one another. Given the width of the absorption features (see Fig. 1), it is therefore likely that the absorption measured at $1033 \mathrm{eV}$ has contributions both from the intense feature at $1031 \mathrm{eV}$ and a transition with different symmetry with an energy around $1033 \mathrm{eV}$.

This means that the model proposed in equations (8) and (9) is incomplete in the sense that it does not consider the overlap between transitions with non-parallel transition dipole moments. The following alternative model is therefore proposed,

$$
\begin{aligned}
& I_{A}=\sigma_{A, 0}+\sigma_{A, 1}^{\prime} \cos ^{2} \vartheta_{A} \sin ^{2} \varphi_{A}+\sigma_{A, 2} \cos ^{2} \vartheta_{B} \sin ^{2} \varphi_{B}+\varepsilon_{A}, \\
& I_{B}=\sigma_{B, 0}+\sigma_{B, 1}^{\prime} \cos ^{2} \vartheta_{B} \sin ^{2} \varphi_{B}+\sigma_{B, 2} \cos ^{2} \vartheta_{A} \sin ^{2} \varphi_{A}+\varepsilon_{B},
\end{aligned}
$$

with the relation between $\vartheta_{A}, \varphi_{A}, \vartheta_{B}, \varphi_{B}$ still dictated according to equation (7).

Fig. 8 shows the fits obtained for each of the experimental distributions, according to the model proposed in equations (12) and (13) and taking $\omega=\pi / 2$. Here, it can be seen that the skews in the distributions of $I_{B}$ are now modeled more accurately than they were using the simpler model (see Fig. 6). The corresponding normalized dichroic model parameters and 
Table 1

Dichroic model parameters, estimated experimental errors, and corresponding uncertainties for the four hyperspectral STXM data sets; all values are normalized such that $\sigma_{A, 0}$ was set to 100 for each data set.

\begin{tabular}{|c|c|c|c|c|c|c|c|c|c|c|c|}
\hline FrMod1 & Value & $u_{P}$ & FrMod2 & Value & $u_{P}$ & FrMod3 & Value & $u_{P}$ & $\mathrm{ZnO}-\mathrm{L}$ & Value & $u_{P}$ \\
\hline$\sigma_{A, 0}$ & 100 & 2.2 & $\sigma_{A, 0}$ & 100 & 2.1 & $\sigma_{A, 0}$ & 100 & 1.7 & $\sigma_{A, 0}$ & 100 & 1.8 \\
\hline$\sigma_{A, 1}^{\prime}$ & 24.7 & 5 & $\sigma_{A, 1}^{\prime}$ & 27.2 & 4 & $\sigma_{A, 1}^{\prime}$ & 21.4 & 3 & $\sigma_{A, 1}^{\prime}$ & 23.5 & 2.8 \\
\hline$\sigma_{A, 2}$ & 5.7 & 4 & $\sigma_{A, 2}$ & 7.1 & 4 & $\sigma_{A, 2}$ & 7.5 & 4 & $\sigma_{A, 2}$ & 3.0 & 3 \\
\hline$\sigma_{B, 0}$ & 71.7 & 1.9 & $\sigma_{B, 0}$ & 68.4 & 1.9 & $\sigma_{B, 0}$ & 71.1 & 1.8 & $\sigma_{B, 0}$ & 72.3 & 3 \\
\hline$\sigma_{B, 1}^{\prime}$ & 16.1 & 3 & $\sigma_{B, 1}^{\prime}$ & 17.0 & 4 & $\sigma_{B, 1}^{\prime}$ & 10.9 & 2.9 & $\sigma_{B, 1}^{\prime}$ & 20.3 & 4 \\
\hline$\sigma_{B, 2}^{D, 1}$ & 14.5 & 4 & $\sigma_{B, 2}^{D, 1}$ & 17.6 & 5 & $\sigma_{B, 2}^{D, 1}$ & 13.4 & 4 & $\sigma_{B, 2}^{D, 1}$ & 16.8 & 3 \\
\hline
\end{tabular}

estimated experimental errors are listed in Tables 1 and 2. The uncertainty in each parameter value is calculated from the previously discussed uncertainties $u_{P \text {, mod }}$ and $u_{P \text {,exp }}$ according to

$$
u_{P}=\left(u_{P, \bmod }^{2}+u_{P, \exp }^{2}\right)^{1 / 2}
$$

\section{Discussion}

From the experimental distributions shown in Fig. 4, it could be observed visually that the distributions obtained from hyperspectral STXM energy stacks are narrower than those obtained from two-energy STXM stacks. Moreover, a difference could be observed between the distributions of $I_{B}$ obtained from the two-energy STXM stack recorded on the ZnO-L and FrMod3 samples [see Fig. 4(d)]. Having modeled these distributions following the XNLD model proposed in
Table 2

Dichroic model parameters, estimated experimental errors, and corresponding uncertainties for the two multispectral STXM data sets; all values were normalized such that $\sigma_{A, 0}$ was set to 100 for both data sets.

\begin{tabular}{llllll}
\hline FrMod3 & Value & $u_{P}$ & ZnO-L & Value & $u_{P}$ \\
\hline$\sigma_{A, 0}$ & 100 & 2.5 & $\sigma_{A, 0}$ & 100 & 2.6 \\
$\sigma_{A, 1}^{\prime}$ & 41.8 & 5 & $\sigma_{A, 1}^{\prime}$ & 42.8 & 4 \\
$\sigma_{A, 2}$ & 14.0 & 4 & $\sigma_{A, 2}$ & 14.2 & 4 \\
$\varepsilon_{A}$ & 4.8 & 0.9 & $\varepsilon_{A}$ & 5.7 & 0.8 \\
$\sigma_{B, 0}$ & 52.8 & 4 & $\sigma_{B, 0}$ & 65.1 & 2.0 \\
$\sigma_{B, 1}^{\prime}$ & 38 & 10 & $\sigma_{B, 1}^{\prime}$ & 21.6 & 4 \\
$\sigma_{B, 2}$ & 31 & 9 & $\sigma_{B, 2}$ & 24.2 & 6 \\
$\varepsilon_{B}$ & 5.6 & 1.1 & $\varepsilon_{B}$ & 5.49 & 0.5 \\
\hline
\end{tabular}

equations (12) and (13), it is now possible to interpret these differences in terms of the dichroic parameters and check their significance based on a statistical hypothesis test. Here, the significance of differences in dichroic parameters was tested using zeta-scores (Analytical Methods Committee, 2016),
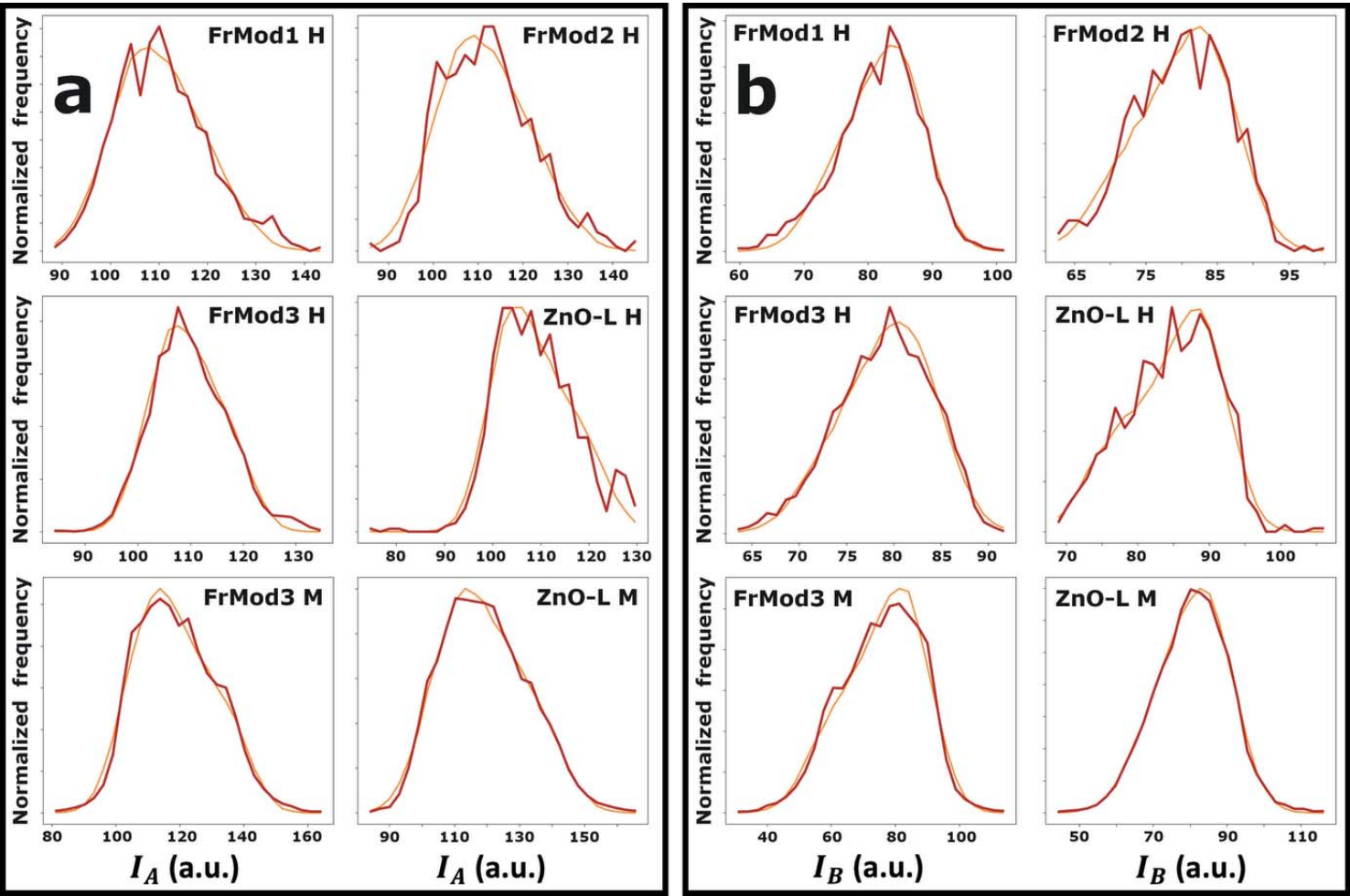

Figure 8

Experimental distributions (red) of $I_{A}(a)$ and $I_{B}(b)$ fitted according to the alternative dichroic model postulated in equations (12) and (13) (orange). 
Table 3

Statistical comparison of the dichroic model parameters $\sigma_{A, 1}^{\prime}$ (left of the diagonal) and $\sigma_{A, 2}$ (right of the diagonal) in terms of $p$-values; all $p$-values lower than 0.05 are highlighted in bold.

\begin{tabular}{lllllll}
\hline & FrMod1 H & FrMod2 H & FrMod3 H & ZnO-L H & FrMod3 M & ZnO-L M \\
\hline FrMod1 H & - & 0.80 & 0.75 & 0.59 & 0.13 & 0.13 \\
FrMod2 H & 0.70 & - & 0.94 & 0.41 & 0.23 & 0.19 \\
FrMod3 H & 0.57 & 0.23 & - & 0.37 & 0.27 & 0.23 \\
ZnO-L H & 0.83 & 0.42 & 0.61 & - & $\mathbf{0 . 0 2 8}$ & $\mathbf{0 . 0 2 8}$ \\
FrMod3 M & $\mathbf{0 . 0 1 6}$ & $\mathbf{0 . 0 2 8}$ & $\mathbf{4 . 7} \times \mathbf{1 0}^{-\mathbf{4}}$ & $\mathbf{1 . 4} \times \mathbf{1 0}^{-\mathbf{3}}$ & - & 0.97 \\
ZnO-L M & $\mathbf{5 . 1} \times \mathbf{1 0}^{-\mathbf{3}}$ & $\mathbf{6 . 9} \times \mathbf{1 0}^{-\mathbf{3}}$ & $\mathbf{1 . 7} \times \mathbf{1 0}^{-\mathbf{5}}$ & $\mathbf{6 . 3} \times \mathbf{1 0}^{-\mathbf{5}}$ & 0.87 & - \\
\hline
\end{tabular}

Table 4

Statistical comparison of the dichroic model parameters $\sigma_{B, 0}$ (left of the diagonal) and $\sigma_{B, 1}^{\prime}$ (right of the diagonal) in terms of $p$-values; all $p$-values lower than 0.05 are highlighted in bold, while $p$ values lower than 0.10 but higher than 0.05 are highlighted in italic.

\begin{tabular}{lllllll}
\hline & FrMod1 H & FrMod2 H & FrMod3 H & ZnO-L H & FrMod3 M & ZnO-L M \\
\hline FrMod1 H & - & 0.86 & 0.23 & 0.40 & $\mathbf{0 . 0 3 6}$ & 0.27 \\
FrMod2 H & 0.23 & - & 0.23 & 0.56 & 0.057 & 0.42 \\
FrMod3 H & 0.82 & 0.32 & - & 0.057 & $\mathbf{9 . 3} \times \mathbf{1 0}^{-\mathbf{3}}$ & $\mathbf{0 . 0 2 8}$ \\
ZnO-L H & 0.87 & 0.27 & 0.73 & - & 0.11 & 0.82 \\
FrMod3 M & $\mathbf{1 . 7} \times \mathbf{1 0}^{-\mathbf{5}}$ & $\mathbf{4 . 7} \times \mathbf{1 0}^{-\mathbf{4}}$ & $\mathbf{2 . 6} \times \mathbf{1 0}^{-\mathbf{5}}$ & $\mathbf{9 . 6} \times \mathbf{1 0}^{-\mathbf{5}}$ & - & 0.13 \\
ZnO-L M & $\mathbf{0 . 0 1 6}$ & 0.23 & $\mathbf{0 . 0 2 8}$ & $\mathbf{0 . 0 4 6}$ & $\mathbf{5 . 1} \times \mathbf{1 0}^{-\mathbf{3}}$ & - \\
\hline
\end{tabular}

found to be significantly lower after excessive exposure of the sample to a tightly focused synchrotron-generated $\mathrm{X}$-ray beam. As X-ray linear dichroism in $\mathrm{ZnO}$ is directly related to the symmetry of its crystal structure, it is hypothesized that the decreased XNLD amplitude is caused by a decrease in the crystallinity of the irradiated crystallites. That is, high $\mathrm{X}$-ray radiation doses are thought to induce a certain level of amorphism which partially breaks the alignment of low-symmetry electronic orbitals. Interestingly, these observations are in direct opposition with the findings of Wang et al. who show that exposing $\mathrm{ZnO}$ nanowires to soft $\mathrm{X}$-rays (O K-edge, $\sim 550 \mathrm{eV}$ ) for several minutes increases the intensity of X-ray induced optical band gap emission with respect to defect-related trap state emission (Wang et al., 2014). The

$$
\zeta=\frac{\left|P_{D}-P_{D^{\prime}}\right|}{\left(u_{P_{D}}^{2}+u_{P_{D^{\prime}}}^{2}\right)^{1 / 2}}
$$

A zeta-score measures the difference between two parameters $P$ of data sets $D$ and $D^{\prime}$ normalized to the expected standard deviation of this difference under the assumption that $P_{D}$ and $P_{D^{\prime}}$ are both measures of the same quantity. For instance, a zeta-score of 1.96 indicates that the difference between $P_{D}$ and $P_{D^{\prime}}$ is equal to two standard deviations, meaning that larger differences are only expected in $5 \%$ of cases $(p=0.05)$.

Tables 3 and 4 show the $p$-values calculated for comparisons of $\sigma_{A, 1}^{\prime}, \sigma_{A, 2}, \sigma_{B, 0}$, and $\sigma_{B, 1}^{\prime}$, based on the values listed in Tables 1 and 2. The other parameters were left out either because they function as normalization factors $\left(\sigma_{A, 0}\right)$, their difference is not relevant $\left(\varepsilon_{A}\right.$ and $\left.\varepsilon_{B}\right)$ or because no statistically significant differences could be found $\left(\sigma_{B, 2}\right)$. It is clear from the tabulated $p$-values that the distributions obtained from the two-energy STXM energy stacks (FrMod3 $\mathrm{M}$ and $\mathrm{ZnO}-\mathrm{L} \mathrm{M}$ ) exhibit XNLD behavior that is significantly different from the distributions obtained from the hyperspectral STXM energy stacks (FrMod1 H, FrMod2 H, FrMod3 $\mathrm{H}$, and $\mathrm{ZnO}-\mathrm{L} \mathrm{H}$ ). These differences are most pronounced in terms of the $\sigma_{A, 1}^{\prime}$ parameter (see Table 3, bottom two rows), which means that significant differences exist in the amplitude of the dichroic effect of the major transition at $1031 \mathrm{eV}$, depending on whether data are retrieved using a high or low radiation dose. Comparing the same data sets, less pronounced statistical differences can also be observed in terms of the $\sigma_{B, 1}^{\prime}$ parameter (see Table 4, final two columns), which measures the amplitude of the dichroic effect of the minor transitions at $1033 \mathrm{eV}$.

Taken altogether, this provides strong evidence that the XNLD behavior of $\mathrm{ZnO}$ is affected by high $\mathrm{X}$-ray radiation doses. To be precise, the amplitude of the XNLD effect was authors ascribe this observation to a form of radiation-induced annealing - in which the energy deposited by the X-rays enables the crystal to cross energy barriers towards energetically more favorable (more crystalline) configurations. It is likely that in the experiments by Wang et al. the radiation dose deposition rate was orders of magnitude lower, producing a distinctly different effect on the material properties of $\mathrm{ZnO}$ than when the dose deposition rate is as high as in the STXM experiments described here.

Focusing on the differences between samples, rather than between measurement methods, two $p$-values do raise some suspicion with regards to the dissimilarity of the $\mathrm{ZnO}-\mathrm{L}$ sample with respect to the three FrMod samples. First, a significant difference $\left(p=5.1 \times 10^{-3}\right)$ in the $\sigma_{B, 0}$ parameter is demonstrated for the distributions obtained on the FrMod3 and $\mathrm{ZnO}$-L samples from two-energy STXM energy stacks. Second, the comparison of the $\sigma_{B, 1}^{\prime}$ parameter for distributions obtained on the FrMod3 and ZnO-L samples from hyperspectral STXM data sets yields a $p$-value of 0.057 . However, since the $\sigma_{B, 0}$ parameter does not measure the amplitude of a dichroic effect and the difference in values of $\sigma_{B, 1}^{\prime}$ is not formally seen as sufficient evidence of dissimilarity, these results are only regarded as pointing in the direction of a possible relation between the production process of $\mathrm{ZnO}$ and its XNLD properties.

This ambiguity in establishing statistically significant dissimilarity between the $\mathrm{ZnO}-\mathrm{L}$ and FrMod points to a major point of potential improvement in the XLD quantification method described here. With the raw data shown in Fig. 4 and the model parameter uncertainties listed in Tables 1 and 2, it is clear that modeling efforts are strongly affected by the low number of analyzed $\mathrm{ZnO}$ crystallites. That is, the fitting of experimental data with model distributions is expected to benefit from including as many crystallite orientations as possible. It is therefore postulated here that the discriminatory 
power of this XLD quantification method can be significantly improved by analyzing larger (or more) crystallite agglomerates per sample.

\section{Conclusion}

It has been shown that it is possible to extract information on the X-ray linear dichroism properties of powders by performing STXM with a nanofocused, linearly polarized X-ray beam. A procedure is proposed that involves (1) the identification of symmetrically distinct diagnostic transitions by means of spectral endmember selection, (2) the modeling of the intensity distributions of the diagnostic transitions, and (3) the estimation of uncertainties in the dichroic model parameters by means of a Monte Carlo simulation. This procedure was demonstrated by analyzing STXM data sets obtained on several differently produced $\mathrm{ZnO}$ powders in both a hyperspectral and multispectral manner. It was found that the intensity distributions at two diagnostic energies could be modeled most accurately by making use of a model that assumes some degree of spectral overlap. Comparing the estimates of the dichroic model parameters for all data sets revealed that samples that received a lower radiation dose (multispectral method) exhibit a more pronounced XNLD effect than those that received a higher radiation dose (hyperspectral method). Moreover, statistical comparison of the XNLD parameters of a lab-synthesized $\mathrm{ZnO}$ sample compared with a commercially produced zinc white point to a possible relationship between the production process of $\mathrm{ZnO}$ and its dichroic properties.

\section{Acknowledgements}

The authors would like to express their gratitude to The Bennink Foundation for funding this research, to Slavica Stankic for providing the $\mathrm{ZnO}-\mathrm{L}$ sample, to Birgit van Driel for providing the FrMod3 sample, to Matthias Alfeld for supplying the SiVM core algorithm, and to Rachid Belkhou and Sufal Swaraj for support at the beamline.

\section{Funding information}

The following funding is acknowledged: The Bennink Foundation.

\section{References}

Analytical Methods Committee (2016). Anal. Methods. 8, 5553-5555. Antel, W. J., Perjeru, F. \& Harp, G. R. (1999). Phys. Rev. Lett. 83, 1439-1442.

Brouder, C. (1990). J. Phys. Condens. Matter, 2, 701-738.

Chiou, J. W., Jan, J. C., Tsai, H. M., Bao, C. W., Pong, W. F., Tsai, M., Hong, I., Klauser, R., Lee, J. F., Wu, J. J. \& Liu, S. C. (2004). Appl. Phys. Lett. 84, 3462-3464.

Fu, J. \& Urquhart, S. G. (2005). J. Phys. Chem. A, 109, 11724-11732.

Gaudry, E., Kiratisin, A., Sainctavit, P., Brouder, Ch., Mauri, F., Ramos, A., Rogalev, A. \& Goulon, J. (2005). Phys. Scr. T115, 10411043.

Goulon, J., Jaouen, N., Rogalev, A., Wilhelm, F., Goulon-Ginet, C., Brouder, C., Joly, Y., Ovchinnikova, E. N. \& Dmitrienko, V. E. (2007). J. Phys. Condens. Matter, 19, 156201.

Hageraats, S., Keune, K., Stankic, S., Stanescu, S., Tromp, M. \& Thoury, M. (2020). J. Phys. Chem. C, 124, 12596-12605.

Juhin, A., Brouder, C., Arrio, M., Cabaret, D., Sainctavit, P., Balan, E., Bordage, A., Seitsonen, A. P., Calas, G., Eeckhout, S. G. \& Glatzel, P. (2008). Phys. Rev. B, 78, 195103.

Kortright, J. B. \& Kim, S. (2000). Phys. Rev. B, 62, 12216-12228.

Kuiper, P., Searle, B. G., Rudolf, P., Tjeng, L. H. \& Chen, C. T. (1993). Phys. Rev. Lett. 70, 1549-1552.

Muller, M. E. (1959). Commun. ACM, 2, 19-20.

Preston, A. R. H., Ruck, B. J., Piper, L. F. J., DeMasi, A., Smith, K. E., Schleife, A., Fuchs, F., Bechstedt, F., Chai, J. \& Durbin, S. M. (2008). Phys. Rev. B, 78, 155114.

Schauries, D., Ney, V., Nayak, S. K., Entel, P., Guda, A. A., Soldatov, A. V., Wilhelm, F., Rogalev, A., Kummer, K., Yakhou, F. \& Ney, A. (2013). Phys. Rev. B, 87, 125206.

Scholl, A., Stohr, J., Luning, J., Seo, J. W., Fompeyrine, J., Siegwart, H., Locquet, J., Nolting, F., Anders, S., Fullerton, E. E., Scheinfein, M. R. \& Padmore, H. A. (2000). Science, 287, 1014-1016.

Schwickert, M. M., Guo, G. Y., Tomaz, M. A., O'Brien, W. L. \& Harp, G. R. (1998). Phys. Rev. B, 58, R4289-R4292.

Smith, A. P. \& Ade, H. (1996). Appl. Phys. Lett. 69, 3833-3835.

Spanke, D., Solinus, V., Knabben, D., Hillebrecht, F. U., Ciccacci, F., Gregoratti, L. \& Marsi, M. (1998). Phys. Rev. B, 58, 5201-5204.

Stöhr, J. (1992). NEXAFS Spectroscopy, edited by G. Ertl, R. Gomer, D. L. Mills and H. K. V. Lotsch, pp. 8-10, pp. 279-280. Berlin: Springer-Verlag.

Thole, B. T., van der Laan, G. \& Sawatzky, G. A. (1985). Phys. Rev. Lett. 55, 2086-2088.

Thurau, C., Kersting, K., Wahabzada, M. \& Bauckhage, C. (2012). Data Min. Knowl. Disc, 24, 325-354.

Wang, Z., Guo, X. \& Sham, T. K. (2014). Nanoscale, 6, 6531-6536.

Zharnikov, M. \& Neuber, M. (2000). Surf. Sci. 464, 8-22. 